This item was submitted to Loughborough's Research Repository by the author.

Items in Figshare are protected by copyright, with all rights reserved, unless otherwise indicated.

\title{
Saltatory waves in the spike-diffuse-spike model of active dendritic spines
}

PLEASE CITE THE PUBLISHED VERSION

LICENCE

CC BY-NC-ND 4.0

\section{REPOSITORY RECORD}

Coombes, S., and P.C. Bressloff. 2019. "Saltatory Waves in the Spike-diffuse-spike Model of Active Dendritic Spines". figshare. https://hdl.handle.net/2134/260. 


\title{
Saltatory waves in the spike-diffuse-spike model of active dendritic spines
}

\author{
S. Coombes ${ }^{1}$ and P. C. Bressloff ${ }^{2}$ \\ ${ }^{1}$ Department of Mathematical Sciences, Loughborough University, Loughborough, LE11 $3 T U$, UK \\ ${ }^{2}$ Department of Mathematics, University of Utah, Salt Lake City, UT 84112
}

(Dated: May 12, 2003)

\begin{abstract}
In this Letter we present the explicit construction of a saltatory traveling pulse of non-constant profile in an idealized model of dendritic tissue. Excitable dendritic spine clusters, modeled with integrate-and-fire (IF) units, are connected to a passive dendritic cable at a discrete set of points. The saltatory nature of the wave is directly attributed to the breaking of translation symmetry in the cable. The conditions for propagation failure are presented as a function of cluster separation and IF threshold.
\end{abstract}

PACS numbers: $87 .^{*}, 05.45 .-\mathrm{a}, 05.45 . \mathrm{Xt}$

The focus of many mathematical studies in physics has been on waves which propagate with constant speed and constant profile. However, there is an increasing body of experimental data from the natural sciences highlighting the existence of waves which travel with non-constant profile. For example, when calcium is released from internal stores into the cytosol of a cardiac myocyte a wave of increased concentration can travel with a lurching quality, where activity is seen to jump from store to store [1]. Another example can be drawn from the field of computational neuroscience where neurons that can fire via post inhibitory rebound are known to underly the generation of lurching waves of activity propagating through an inhibitory network [2]. Such lurching waves are typically referred to as saltatory. In this Letter we present the explicit construction of a saltatory wave in an idealized model of a neuronal dendrite.

In the cerebral cortex approximately $80 \%$ of all excitatory synapses are made onto dendritic spines. These are small mushroom like appendages with a bulbous head and a tenuous stem (of length around $1 \mu \mathrm{m}$ ) and may be found in their hundreds of thousands on the dendritic tree of a single cortical pyramidal cell. The biophysical properties of spines have been linked with mechanisms for Hebbian learning [3], the implementation of logical computations [4], coincidence detection [5], orientation tuning in complex cells of visual cortex [6], the amplification of distal synaptic inputs [7]. The implication of excitable channels in the spine head membrane for amplification of excitatory synaptic inputs was first discussed by Jack et al. [8]. However, it is only relatively recently that confocal and two-photon microscopy observations have confirmed the generation of action potentials in the dendrites. Since dendritic spines possess excitable membrane, the spread of current from one spine along the dendrites may bring adjacent spines to threshold for impulse generation, resulting in a saltatory propagating wave in the distal dendritic branches [9].

The first theoretical study of wave propagation mediated by dendritic spines was carried out by Baer and Rinzel [10]. They considered a continuum model of a den-

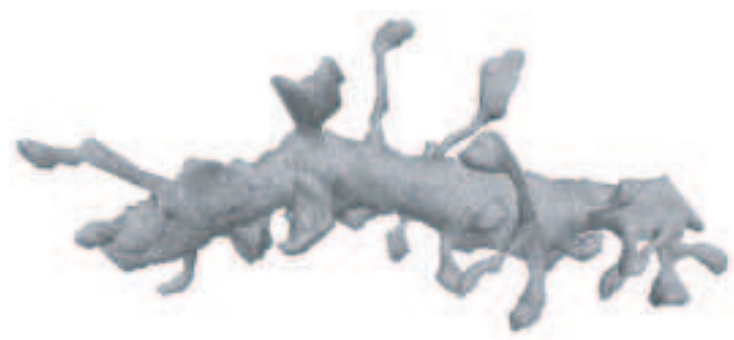

FIG. 1: An example of a piece of spine studded dendritic tissue (from rat hippocampal region CA1 stratum radiatum) $\sim 5 \mu \mathrm{m}$ in length. Taken with permission from Synapse Web, Boston University, http://synapses.bu.edu

dritic tree coupled to a distribution of excitable dendritic spines. The active spine head dynamics is modeled with Hodgkin-Huxley kinetics whilst the (distal) dendritic tissue is modeled with the cable equation. The spine head is coupled to the cable via a spine stem resistance that delivers a current proportional to the number of spines at the contact point. There is no direct coupling between neighboring spines; voltage spread along the cable is the only way for spines to interact. Numerical studies of the Baer-Rinzel model [10] show both smooth and saltatory traveling wave solutions, the former arising in the case of uniform spine distributions and the latter when spines are clustered in groups. The saltatory nature of a propagating wave may be directly attributed to the fact that active spine clusters are physically separated. In this paper we present an alternative, analytically tractable treatment of saltatory waves based on the so-called spike-diffuse-spike (SDS) model of active dendritic spines [11, 12]. The SDS model, which reduces the spine head dynamics to an allor-nothing action potential response, was previously used to construct exact solutions for smooth waves in the case of a uniform spine density. However, this analysis was limited since it did not capture the true saltatory nature of a dendritic wave. Here we explicitly take into account the discrete nature of spine clusters, and explicitly construct the corresponding saltatory waves. We also derive dispersion curves for the speed of the wave as a function of cluster spacing and the spine threshold, and determine 
the conditions for wave propagation failure.

Let $\rho(x)$ represent the spine density per unit length along a uniform, passive dendritic cable. Denoting the voltage at position $x$ on the cable at time $t$ by $V=$ $V(x, t)$, the associated cable equation is given by

$$
\tau \frac{\partial V}{\partial t}=-V+\lambda^{2} \frac{\partial^{2} V}{\partial x^{2}}+\lambda^{2} r_{a} \rho(x) \frac{\widehat{V}-V}{r},
$$

where $\tau$ and $\lambda$ are the membrane time constant and the electronic space constant of the cable. The parameter $r$ is the spine stem resistance of an individual spine and $r_{a}$ is the intracellular resistance per unit length of cable. In the SDS model, the function $\widehat{V}(x, t)$ represents the sequence of action potentials generated in the spine head at $x$ whenever the associated subthreshold spine head potential $U(x, t)$, driven by current from the shaft, crosses some threshold $h$. Given the high resistance of the spine stem, we neglect subthreshold currents into the cable. The voltage $U$ evolves according to the integrateand-fire (IF) equation

$$
\widehat{C} \frac{\partial U}{\partial t}=-\frac{U}{\widehat{r}}+\frac{V-U}{r},
$$

such that whenever $U$ crosses the threshold $h$ it is immediately reset to zero. Here $\widehat{C}$ and $\widehat{r}$ are the membrane capacitance and resistance of the spine head. Let $t^{j}(x)$ denote the $j$ th firing time of the spine head at position $x$ such that $U\left(x, t^{j}(x)\right)=h$. Then $\widehat{V}(x, t)=\sum_{j} \eta\left(t-t^{j}(x)\right)$ with $\eta(t)=0$ for all $t<0$. The shape of the action potential is specified by the function $\eta(t)$, which can be fitted to the universal shape of an action potential.

In the original formulation of the SDS model, the spine density function was taken to be uniform. Although impulse propagation failure is known to occur if the spine density is below some critical level, the numerical studies of Baer and Rinzel suggest that propagation may be recovered by redistributing the spines into equally spaced dense clusters. Since interspine distances are of the order of $\mu \mathrm{m}$ and electronic length is typically measured in $\mathrm{mm}$ we shall consider spine head voltage at a cluster site to be the local spatial average of membrane potential in adjacent spines. Hence, we consider a discrete distribution of spines for which $\rho(x)=\bar{n} \sum_{m} \delta\left(x-x_{m}\right)$ where $x_{m}$ is the location of the $m$ th spine cluster and $\bar{n}$ is the number of spines in a cluster. Such a distribution breaks continuous translation symmetry so that saltatory or lurching waves are expected rather than traveling waves of constant profile. We define a saltatory wave as an ordered sequence of firing times $\ldots t_{m-1}<t_{m}<t_{m+1}$ in which each spine cluster only fires once. The corresponding set of threshold conditions is $U\left(x_{m}, t_{m}\right)=h$ for all $m$ with $U\left(x_{m}, t\right)$ for $t \leq t_{m}$ obtained by integrating equation (2) from $(-\infty, t)$ using the initial condition $\lim _{t \rightarrow-\infty} V(x, t)=0$ :

$$
U\left(x_{m}, t\right)=\frac{1}{\widehat{C} r} \int_{-\infty}^{t} \mathrm{e}^{\varepsilon_{0}(s-t)} V\left(x_{m}, s\right) \mathrm{d} s,
$$

where $\varepsilon_{0}=(1 / r+1 / \widehat{r}) / \widehat{C}$. Under the approximation $\lambda^{2} r_{a} \bar{n} / r \ll 1$, equation (1) may be solved as

$$
V(x, t)=\frac{\bar{n} D r_{a}}{r} \sum_{m} H\left(x-x_{m}, t-t_{m}\right),
$$

where $D=\lambda^{2} / \tau$ is the diffusion coefficient for the cable and

$$
H(x, t)=\int_{0}^{t} G(x, t-s) \eta(s) \mathrm{d} s .
$$

The Green's function in equation (5) is that of the uniform cable equation:

$$
G(x, t)=\frac{\mathrm{e}^{-t / \tau}}{\sqrt{4 \pi D t}} \mathrm{e}^{-x^{2} /(4 D t)} \Theta(t),
$$

where $\Theta(t)$ is the Heaviside step function. Equation (4) is formally equivalent to the solution of the fire-diffuse-fire model of $\mathrm{Ca}^{2+}$ release [13], although in the latter model firing times are not generated by an IF process.

Suppose that the spine clusters are uniformly distributed along the cable such that $x_{m}=m d$ where $d$ is the spacing between clusters. Equation (4) then has a saltatory wave solution of the form $t_{m}=m \Delta$. The parameter $\Delta$ measures the time between successive threshold crossings at adjacent spine heads such that the speed $v$ of threshold crossing events is $d / \Delta$. The speed is determined from the threshold condition $U(m d, m \Delta)=h$, which is independent of $m$. In order to calculate the wave speed, we first Fourier transform equation (4) with respect to $x$ and $t$ using the inverse transforms

$$
\begin{gathered}
\eta(t)=\int_{-\infty}^{\infty} \mathrm{e}^{i \omega t} \widetilde{\eta}(\omega) \frac{d \omega}{2 \pi}, \\
G(x, t)=\int_{-\infty}^{\infty} \mathrm{e}^{i k x} \mathrm{e}^{-\sigma(k) t} \frac{d k}{2 \pi}
\end{gathered}
$$

where $\sigma(k)=\varepsilon+D k^{2}, \varepsilon=\tau^{-1}$. This gives

$$
\begin{aligned}
V(x, t)= & \frac{\bar{n} D r_{a}}{r} \int_{-\infty}^{\infty} \mathrm{e}^{i \omega t} \frac{d \omega}{2 \pi} \int_{-\infty}^{\infty} \mathrm{e}^{i k x} \frac{d k}{2 \pi} \widetilde{G}(k, \omega) \widetilde{\eta}(\omega) \\
& \times \sum_{m} \mathrm{e}^{-i(k d+\omega \Delta) m}
\end{aligned}
$$

with $\widetilde{G}(k, \omega)=1 /(i \omega+\sigma(k))$. We now use the identity

$$
\sum_{m} \mathrm{e}^{-i m(k d+\omega \Delta)}=\frac{2 \pi}{d} \sum_{p} \delta(k+\omega / v-2 \pi p / d),
$$

to eliminate the $k$-integral:

$$
\begin{aligned}
V(x, t) & =\frac{\bar{n} D r_{a}}{r d} \sum_{p} \mathrm{e}^{2 \pi i p x / d} \times \\
& \int_{-\infty}^{\infty} \frac{d \omega}{2 \pi} \frac{\widetilde{\eta}(\omega) e^{i \omega(t-x / v)}}{i \omega+\sigma(2 \pi p / d-\omega / v)} .
\end{aligned}
$$




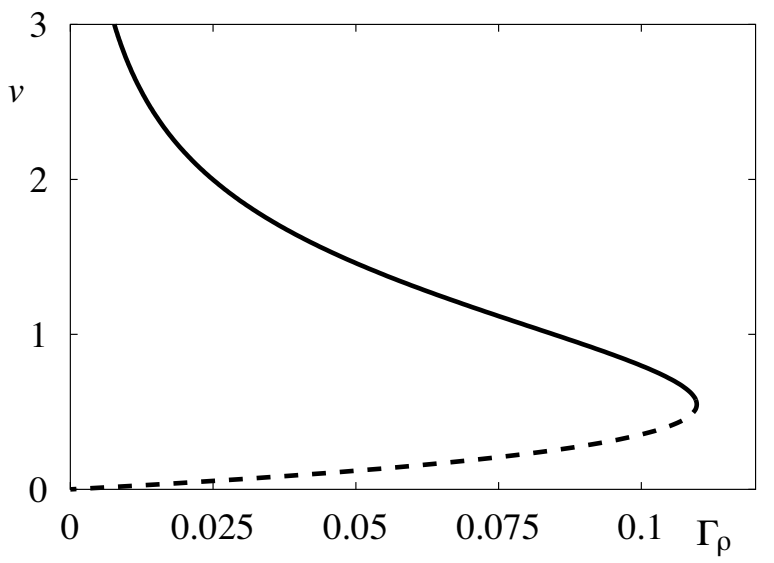

FIG. 2: A plot of wave speed $v$ as a function of threshold $\Gamma_{\rho}$, as determined by equation (13) for the continuum SDS model. Here $\varepsilon=\varepsilon_{0}=\tau_{R}=D=1$. The upper (lower) branch is stable (unstable).

In this representation it is clear that the $V(x, t)$ is invariant under the combined translation $(x, t) \rightarrow(x+d, t+\Delta)$.

It is useful to consider here the continuum limit in which $d \rightarrow 0$ such that the total number of spines remains fixed. That is, we fix $\bar{n} / d=\bar{\rho}$ independent of $d$. In this case only the term with $p=0$ contributes to (11) and the threshold condition becomes

$$
h=\frac{\bar{\rho} D r_{a}}{\widehat{C} r^{2}} \int_{-\infty}^{\infty} \frac{\mathrm{d} \omega}{2 \pi} \frac{\widetilde{\eta}(\omega)}{[\sigma(\omega / v)+i \omega]\left[\varepsilon_{0}+i \omega\right]} .
$$

This integral can be evaluated by closing the contour in the lower-half complex plane. Since $\eta(t)=0$ for $t<0$ it follows that any poles of $\widetilde{\eta}(\omega)$ lie in the upper-half complex plane so that we only have to consider poles arising from the zeros of the function $\sigma(\omega / v)+i \omega$. The latter are given by $\omega=i \omega_{ \pm}$where $\omega_{ \pm}=v\left[-v \pm \sqrt{v^{2}+4 \varepsilon D}\right] /(2 D)$. Hence the wave speed satisfies

$$
\widetilde{h}=\frac{\bar{\rho} v^{2}}{D} \frac{\widetilde{\eta}\left(i \omega_{-}\right)}{\left[\omega_{+}-\omega_{-}\right]\left[\varepsilon_{0}-\omega_{-}\right]},
$$

where $\widetilde{h} \equiv h \widehat{C} r^{2} /\left(D r_{a}\right)$. This is an implicit expression for $v$ as a function of system parameters. As a concrete example consider the shape of the action potential to be a rectangular pulse of strength $\eta_{0}$ and duration $\tau_{R}$. In this case we write $\eta(t)=\eta_{0} \Theta(t) \Theta\left(\tau_{R}-t\right)$, for which $\widetilde{\eta}(\omega)=$ $\eta_{0}\left(1-\mathrm{e}^{i \omega \tau_{R}}\right) / i \omega$. A plot of wave speed $v=v\left(\Gamma_{\rho}\right)$ as a function of the rescaled threshold $\Gamma_{\rho}=\widetilde{h} /\left(\eta_{0} \bar{\rho}\right)$ is shown in Fig. 2. A fast and slow wave are found to coalesce in a saddle node bifurcation, illustrating that above some critical threshold $h$ or below some critical spine density $\bar{\rho}$ solitary pulses fail to propagate. The stability theory for the continuum case has been developed previously [11] and may be used to show that it is the faster of the two branches that is stable.

An alternative way to calculate (4), practical for numerically obtaining results for finite $d$, is to use (8) and write (5) in the form

$$
H(x, t)=\int_{-\infty}^{\infty} \frac{\mathrm{d} k}{2 \pi} \mathrm{e}^{i k x} \mathrm{e}^{-\sigma(k) t} \eta(k, t),
$$

where $\eta(k, t)=\int_{0}^{t} \eta(s) \mathrm{e}^{\sigma(k) s} \mathrm{~d} s$. One may then exploit the convolution structure of (14) to evaluate it in closed form for a given $\eta(t)$. In the interests of brevity and clarity of exposition we choose instead to focus on our previous choice of rectangular pulse. In this case $\eta(k, t)=\eta_{0}\left(\exp \left(\sigma(k) \min \left(t, \tau_{R}\right)\right)-1\right) / \sigma(k)$, so that $H(x, t)=A_{\varepsilon}\left(x, t-\min \left(t, \tau_{R}\right)\right)-A_{\varepsilon}(x, t)$, with

$$
A_{\varepsilon}(x, t)=\eta_{0} \int_{-\infty}^{\infty} \frac{\mathrm{d} k}{2 \pi} \frac{\mathrm{e}^{i k x-\sigma(k) t}}{\sigma(k)} .
$$

This is a standard integral [14] given explicitly by

$$
\begin{aligned}
& \frac{\eta_{0}}{4} \sqrt{\frac{1}{\varepsilon D}}\left\{\exp \left(-|x| \sqrt{\frac{\varepsilon}{D}}\right) \operatorname{erfc}\left(-\frac{|x|}{\sqrt{4 D t}}+\sqrt{\varepsilon t}\right)\right. \\
& \left.+\exp \left(|x| \sqrt{\frac{\varepsilon}{D}}\right) \operatorname{erfc}\left(\frac{|x|}{\sqrt{4 D t}}+\sqrt{\varepsilon t}\right)\right\} .
\end{aligned}
$$

Using this approach means that we may write the threshold condition in the form

$$
\widetilde{h}=\bar{n} \sum_{m=1}^{\infty} \widehat{H}(m d, m \Delta)
$$

where $\widehat{H}(x, t)=\widehat{A}\left(x, t-\tau_{R}\right)-\widehat{A}(x, t)$, and

$$
\widehat{A}(x, t)=\int_{-\infty}^{0} \mathrm{e}^{\varepsilon_{0} s} A_{\varepsilon}(x, t-s) \mathrm{d} s .
$$

Using (15) it may be shown that $\widehat{A}(x, t)=\left(A_{\varepsilon}(x, t)-\right.$ $\left.A_{\varepsilon+\varepsilon_{0}}(x, t)\right) / \varepsilon_{0}$. The sum in (17) can then be performed numerically to obtain the speed of a lurching wave $v=$ $v\left(d, \Gamma_{n}\right)$ where $\Gamma_{n}=\widetilde{h} /\left(\eta_{0} \bar{n}\right)=\Gamma_{\rho} / d$.

In Fig. 3 we plot the speed $v$ as a function of cluster spacing $d$ for fixed threshold $\Gamma_{n}$, which shows that if the spine clusters are separated beyond some critical spacing, on the order of the electronic length, a saltatory pulse will fail to propagate. By generalizing the stability analysis in [14] it is possible to establish that, as in the continuum model, it is the faster of the two branches that is stable. It is also instructive to consider the region in the $\left(d, \Gamma_{n}\right)$ parameter plane where saltatory pulses exist. This may be obtained by continuing the limit point defining propagation failure of a saltatory pulse in the $(d, v)$ plane as a function of $\Gamma_{n}$. The resulting phase diagram is shown in Fig. 4, and establishes that with increasing $d$ the critical threshold for propagation failure decreases. Interestingly the minimum wave speed of a stable saltatory pulse is found to be relatively insensitive to variation in cluster spacing $d$ and threshold $\Gamma_{n}$. In physical units of $\mathrm{ms}^{-1}$ this speed is $O(1)$ 


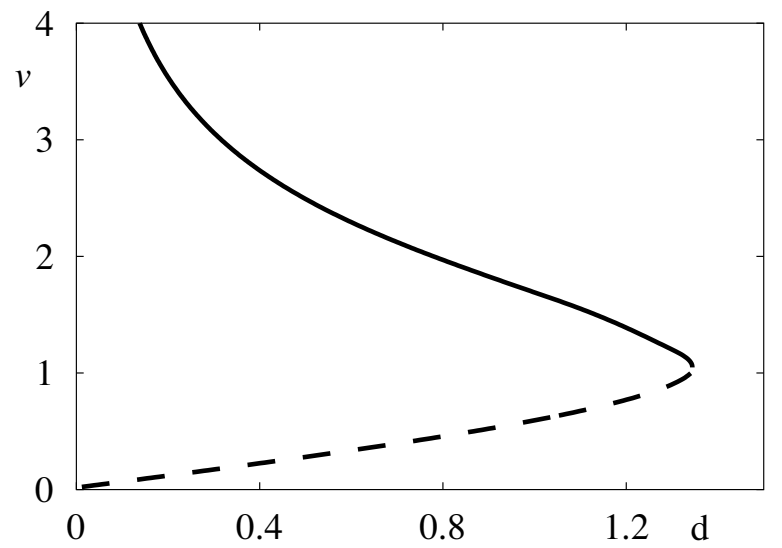

FIG. 3: A plot of wave speed $v$ for a saltatory pulse as a function of cluster spacing $d$. Here, $\Gamma_{n}=0.05$ and $\varepsilon=\varepsilon_{0}=$ $\tau_{R}=D=1$. The upper (lower) branch is stable (unstable).

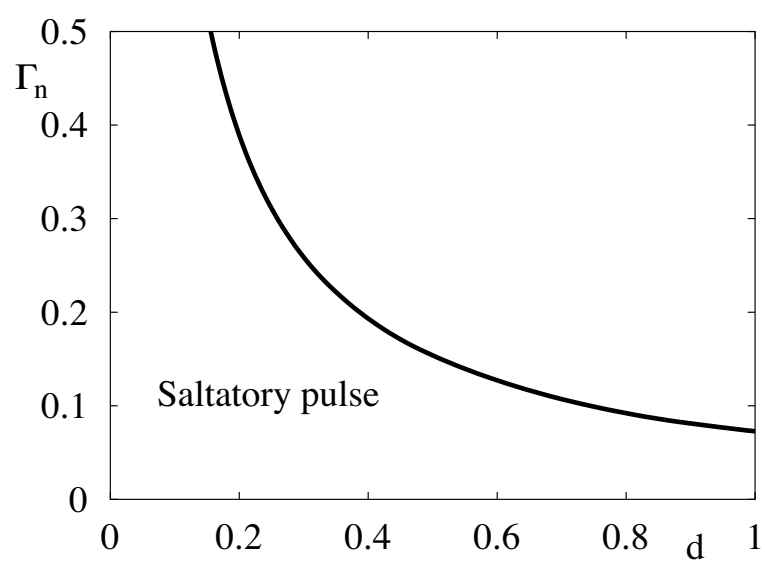

FIG. 4: Continuation of the limit point in Fig. 3 showing the region in $\left(d, \Gamma_{n}\right)$ where stable saltatory traveling waves exist.

which is consistent with experimentally measured dendritic spike speeds in cortical pyramidal cells [15]. Now that we have obtained the speed of a saltatory pulse as a function of system parameters it is possible to close our expression for the shape of a solitary pulse given by $V(x, t)=\bar{n} D r_{a} / r \sum_{m} H(x-m d, t-m d / v)$. A plot of this analytical expression is shown in Fig. 5, which clearly demonstrates that the saltatory pulse has a non-constant profile.

In essence the work in this paper shows that the SDS model is ideal for analytically exploring aspects of dendritic function known to be subserved by active spines. One natural extension of our work is to explore the scattering of saltatory waves in branched structures by considering branching nodes with boundary conditions that ensure continuity of potential and conservation of current. Another natural extension is to treat noise at the level of spines using techniques recently developed for the study of clusters of stochastic receptor channels [16].

From a more general perspective, the use of physi-

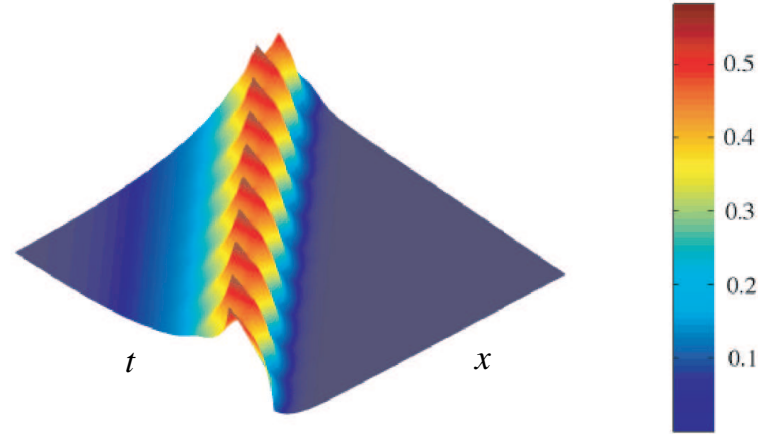

FIG. 5: Plot of the analytically obtained saltatory solution $V(x, t)$ in the dendritic cable with parameters as in Fig. 3 and $d=1$. The $x$ axis covers 10 lattice sites and the $t$ axis $10 d / v$.

cally separated excitable units, each capable of generating an elementary signal, and embedded in a diffusive medium is likely to be a universal biological mechanism for metabolically efficient signaling. In the case of neurons the excitable unit is a cluster of spines, the signal a calcium spike, and the diffusive medium the dendrite. In the context of intracellular calcium signaling we may replace spine by calcium sensitive receptor, spike by puff (describing the calcium released from stores in the endoplasmic reticulum) and dendrite by cytosol.

[1] N. Callamaras, J. S. Marchant, X. P. Sun, and I. Parker, Journal of Physiology 509, 81 (1998).

[2] J. Rinzel, D. Terman, X. J. Wang, and B. Ermentrout, Science 279, 1351 (1998).

[3] A. Zador, C. Koch, and T. H. Brown, Proceedings of the National Academy of Sciences USA 87, 6718 (1990).

[4] G. M. Shepherd and R. K. Brayton, Neuroscience 21, 151 (1987).

[5] M. E. Larkum, J. J. Zhu, and B. Sakmann, Nature 398, 338 (1999).

[6] B. W. Mel, D. L. Ruderman, and K. A. Archie, The Journal of Neuroscience 18, 4325 (1998).

[7] J. P. Miller, W. Rall, and J. Rinzel, Brain Research 325, 325 (1985).

[8] J. J. B. Jack, D. Noble, and R. W. Tsien, Electric Current Flow in Excitable Cells (Clarendon Press, 1975).

[9] G. M. Shepherd, R. K. Brayton, J. P. Miller, I. Segev, J. Rinzel, and W. Rall, Proceedings of the National Academy of Sciences USA 82, 2192 (1985).

[10] S. M. Baer and J. Rinzel, Journal of Neurophysiology $\mathbf{6 5}$, 874 (1991).

[11] S. Coombes and P. C. Bressloff, SIAM Journal on Applied Mathematics 61, 432 (2000).

[12] S. Coombes, Mathematical Biosciences 170, 155 (2001).

[13] J. E. Keizer, G. D. Smith, S. Ponce Dawson, and J. Pearson, Biophysical Journal 75, 595 (1998).

[14] S. Coombes, Bulletin of Mathematical Biology 63, 1 (2001).

[15] G. Buzsaki and A. Kandel, Journal of Neurophysiology 79, 1587 (1998).

[16] J. W. Shuai and P. Jung, Physical Review E 67, 031905 (2003). 